\title{
Sexually transmitted diseases amongst pregnant Vietnamese refugees in Hong Kong
}

\author{
P A King, S J Duthie, H K Ma
}

\begin{abstract}
In a prospective study the prevalence of syphilis amongst a group of pregnant Vietnamese refugees in Hong Kong was found to be $3.4 \%$. No cases of gonorrhoea, genital warts or genital ulcers were found in the same group of patients. The implications of these results are discussed and the importance of adequate screening, treatment, contact tracing and health education amongst this group are emphasised.
\end{abstract}

A previous retrospective study of the obstetric and perinatal outcome amongst Vietnamese refugees in Hong Kong reported a high prevalence of syphilis in this antenatal population when compared wth an age-matched Chinese control group. ${ }^{1}$ A prospective study was conducted to confirm this observation and to determine the coincidental occurrence of gonorrhoea in the same population.

\section{Patients and methods}

From February 1989 to May 1989 (inclusive) all Vietnamese refugees attending the antenatal booking clinic were screened for syphilis and gonorrhoea. The Venereal Diseases Research Laboratory (VDRL) test was used as the initial screening test for syphilis. If positive, additional confirmation was obtained by the Fluorescent Treponemal Antibody Absorption (FTA Abs) test. A diagnosis of syphilis was made on serological grounds if the FTA (ABS) test was positive and if the VDRL titre rose between two consecutive tests. Women with positive serological tests for syphilis had a careful clinical examination seeking stigmata of syphilis or tissue paper scarring of the skin which occurs in yaws,

\footnotetext{
Department of Obstetrics and Gynaecology, University of Hong Kong, Tsan Yuk Hospital, Hospital Road, Hong Kong

P A King, S J Duthie, H K Ma
}

and were then referred to the social hygiene clinic for appropriate management.

During the initial examination a speculum examination was performed and a conscious effort was made to look for genital tract ulceration and genital warts. A cervical smear was sent to the regional cytology laboratory. Endocervical and urethral swabs were taken from each patient and immediately innoculated on to Thayer Martin medium. The plates were taken promptly to the laboratory for incubation and were processed by standard microbiological techniques.

\section{Results}

A total of 179 Vietnamese refugees were screened during this period. There was no evidence of genital tract ulceration or macroscopic condyloma in any of the women. The cervical smears were all negative for dysplastic cells and koilocytes. Six women $(3.4 \%)$ had serological evidence of syphilis. There was one biological false positive VDRL result. Urethral and endocervical swabs for gonorrhoea were available in 158 of the patients, including those with positive serology, and there were no positive results. Gonorrhoea cultures were not taken from 21 women because either culture medium was not available or the investigators were unavailable to take the necessary specimens.

\section{Discussion}

The striking finding of this study is the high prevalence of syphilis and the absence of gonorrhoea amongst pregnant Vietnamese refugees in Hong Kong.

Data on the prevalence of sexually transmitted diseases in Vietnam are virtually non-existent in the Western literature. Thus it is impossible to compare the prevalence of syphilis in this refugee population with that of the general population in Vietnam. The prevalence of syphilis in this group is several times higher than that reported from industrialised countries such as the United Kingdom, the USA and the German Democratic 
Republic. $^{23}$ In Hong Kong, which is a newly industrialised country, syphilis has become increasingly rare in the general population. ${ }^{4}$

It is a generally held view that syphilis is more common in developing countries. Reports of treponemal seroreactor rates in the third world range from $1.9 \%$ among antenatal patients in Nigeria to $94 \%$ among prostitutes in Agra, India. ${ }^{5}$ We speculate that the prevalence of syphilis amongst the Vietnamese refugees in Hong Kong reflects that in Vietnam. The mass migration of these refugees to Hong Kong serves as a clear reminder that syphilis is not a ghost of the past.

The ubiquitous gonococcus is one of the most common communicable pathogens in developed countries and the available data indicate that its occurrence is even more common in developing countries. ${ }^{5}$ However, in this group of patients there was not a single case of gonorrhoea. It is unlikely that patients with gonorrhoea were undetected since specimen collection, inoculation and incubation were carried out under optimal conditions. Is it possible that these Vietnamese patients have yaws rather than syphilis? We consider this unlikely for several reasons. No patient had tissue paper scarring of the skin. ${ }^{6}$ During the period of the study a Vietnamese refugee was diagnosed as having secondary syphilis by the appearance of the maculo-papular rash of syphilis with palmar syphylides and positive serology. Another refugee was admitted as an emergency and delivered a macerated stillbirth at 32 weeks gestation. The necropsy showed numerous spirochaetes in the fetal liver. Neither of these patients is in our prospective study because they had not booked for antenatal care. Nonetheless these cases illustrate, in a graphic and tragic manner, that the complications of syphilis do occur in this population.

The interpretation of anti-treponemal antibody tests can be difficult and it is possible that these patients have non-venereal endemic treponemal disease rather than venereal syphilis. The implications for clinical management are similar as the risk to the fetus in pregnant patients with syphilis are well documented. ${ }^{7-9}$ Syphilis during pregnancy must be adequately diagnosed and treated both to prevent the complications of late abortion, still birth and congenital syphilis and for the future health of the mother.

Another possible explanation for the lack of gonorrhoea is that patients may have contracted both syphilis and gonorrhoea and been treated with drugs other than penicillin such as kanamycin or spectinomycin. These drugs will not abort coincident incubating syphilis. ${ }^{5}$ Owing to the language barrier and the absence of personal medical records this speculation is difficult to substantiate.

Two fundamental principles in the public health control of syphilis are contact tracing and education. It is alarming that in Hong Kong facilities for contact tracing within the refugee camps do not exist. There is an obvious risk of reinfection and development of the long term complications of syphilis which include destructive lesions of the cardiovascular and nervous systems. ${ }^{5}$ Syphilis tends to be more common amongst men than women ${ }^{10}$ and we postulate that a reservoir of infection exists in the refugee camps. These observations are important especially to health care personnel in host countries where refugees ultimately settle. There is also a dearth of education in this group. Ideally the treatment of an episode of sexually transmitted disease should be used as an opportunity for health education. Unfortunately there is a shortage of skilled interpreters and other priorities such as basic health care, education of children and family planning advice compete for the finite resources which are available.

In summary, we would like to emphasise that positive syphilis serology is common among pregnant Vietnamese refugees in Hong Kong. Screening, adequate treatment, contact tracing and patient education are all important aspects of management which are essential to check the spread of treponemal diseases and to prevent their late complications.

1 King PA, Duthie SJ, Li DFH, Ma HK. Obstetric and perinatal outcome amongst Vietnamese refugees in Hong Kong: An age-matched case-controlled study. Int J Gynaecol Obstet. (In press.)

2 WHO Expert Committee on Venereal Diseases and Treponematoses. Sixth Report. World Health Organisation. 1986. Technical Report Series 736. Geneva; p.26.

3 Communicable Disease Surveillance Centre. Sexually transmitted disease surveillance in Britain: 1983. Br Med J 1985;291:528-9.

4 Medical and Health Department. Annual Departmental Reports 1980-1988. Government printer, Hong Kong.

5 Arya OP, Osoba AO, Bennett FJ. Tropical Venereology. Edinburgh: Churchill Livingstone, 1988.

6 Sparling PF. Natural history of syphilis. In: Holmes KK, Mardh P-A, Sparling PF, Weisner PJ, eds. Sexually Transmitted Diseases. New York: McGraw Hill, 1984:298-305.

7 Mascola L, Pelosi R, Blount JH, Binkin NJ, Alexander CE, Cates W Jr. Congenital Syphilis. Why is it still occurring? JAMA 1984;252:1719-22.

8 Anonymous. Yaws or syphilis? Br Med J 1979;283:912.

9 Duthie SJ, King PA, Yung GLK, Ma HK. Routine serological screening for syphilis during pregnancy-disposable anachronism or fundamental necessity? Aust NZ J Obstet Gynaecol 1990;30:29-31.

10 Jeyasingh P, Ramanaiah TBBSV, Fernandes SD. Pattern of sexually transmitted diseases in Madurai, India. Genitourin Med 1985;61:399-403.

Accepted for publication 24 April 1990. 